\title{
TOWARDS REQUIREMENTS ELICITATION IN SERVICE- ORIENTED BUSINESS NETWORKS USING VALUE AND GOAL MODELLING
}

\author{
Rodrigo Mantovaneli Pessoa, Marten van Sinderen \\ University of Twente, 7500 AE Enschede, The Netherlands \\ \{r.mantovanelipessoa,m.j.vansinderen\}@ewi.utwente.nl \\ Dick Quartel \\ Novay, 7500 AN Enschede, The Netherlands \\ Dick.Quartel@novay.nl
}

\begin{abstract}
Keywords: Requirements elicitation, value modelling, goal modelling, coordination process modelling, service-oriented architecture.

Abstract: Due to the contemporary trends towards increased focus on core competences and outsourcing of non-core activities, enterprises are forming strategic alliances and building business networks. This often requires cross enterprise interoperability and integration of their information systems, leading to widespread adoption of Service Oriented Architectures (SOA). In this paper we present an approach to guide the development and evolution of service-oriented business networks. Our approach combines value models and goal models, where the former are used to represent and analyse the economic sustainability of a business network and the latter are used to represent and analyse the goals of the participants within the business network. Systematic guidelines are proposed to derive a goal model from a value model. In addition, a preliminary discussion is presented on how to refine goals and operationalize goals as services rooted in a SOA. The approach is illustrated using an example of a business network in the electricity sector.
\end{abstract}

\section{INTRODUCTION}

In order to improve their efficiency and better meet customer needs, many enterprises organize themselves as networked business constellations, called business webs (Tapscott et al. 2000) or business networks. Business networks are formed by profit-and-loss responsible business units, or independent companies, that have decided to cooperate in producing and consuming goods and services for achieving specific purposes. For this, the participants in a business network should be able to to sustain economic activity by exchanging items of value against money, while operating according to their respective business goals with proper focus on core competencies. The operational fulfilment of business networks usually requires cross-enterprise interoperability and integration of information systems (Mantovaneli Pessoa et al. 2008, Quartel et al. 2008), which can be facilitated by the adoption of Service-Oriented Architectures (SOA) (OASIS 2006).

The SOA paradigm endorses services as the fundamental design concept for developing software architectures and shifts developer focus from the confines of an individual application towards an open market of services hosted on arbitrary computing platforms in a distributed environment. SOA-compliant (web services) standards not only allow the definition of information exchange (e.g., XML, WSDL, SOAP) but also of the coordination and composition of services (e.g., BPEL). Thus, in the SOA paradigm, services perform functions implemented in software, exposed by well-defined interfaces which provide the mechanism by which services can communicate with one another in compositions to perform higher level functionality. Although the claimed benefits of SOA are manifold (Papazoglou \& Ribbers 2006), there is no evidence 
that the mere adoption of SOA will automatically lead to improved business networks or agility of the participants in such networks. An important challenge, therefore, is to enable consideration of business factors (Luthria \& Rabhi 2009) and enforce fulfilment of corresponding requirements when developing technical SOA-based solutions.

In this paper, we propose an approach that addresses complementary business concerns as a foundation for designing service-oriented business networks, i.e., business networks with technical support rooted in SOA. These concerns are economic sustainability, goal fulfilment, and process coordination, which are respectively expressed and analysed using value, goal and process models. Our focus in this paper will be on the development of a goal model based on a given value model.

The paper is further structured as follows. Section 2 summarizes our overall approach. Section 3 presents some related work. Section 4 describes our main contribution, namely initial guidelines for the development of a goal model from a given value model. This section uses a running example to illustrate our approach. Section 5 briefly discusses how to map goals onto services that can be operationalized in a SOA. Finally, Section 6 presents our conclusions and identifies further research.

\section{OVERALL APPROACH}

Business networks are complex systems, hence creating models of such systems in order to understand and define them normally requires a multi-viewpoint approach in which different concerns and interests are distinguished and captured by separate models. An important consequence of this 'divide and conquer' strategy is that in any individual development project proper attention should be given to the mutual consistency of the various models (Pijpers \& Gordijn 2008, Dijkman et al. 2008), since they all refer to the same phenomenon (i.e., a business network). In our approach, we focus on goal, value and process models (see Fig. 1).

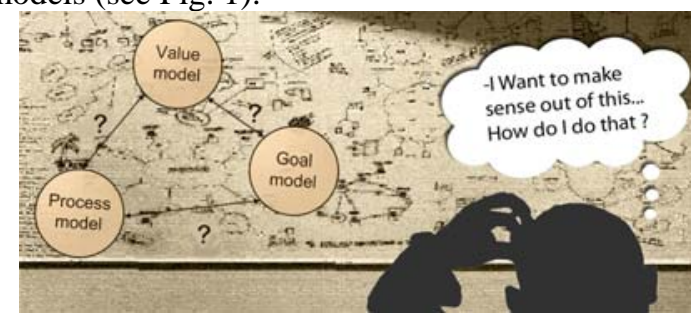

Figure 1: Different perspectives of enterprise architectures require different models.
A value model is used to represent that something of economic value is exchanged; a goal model focuses on describing intentional aspects of business activities; and a process model typically shows what these activities are and how they are coordinated.

Our proposal is to initially design and analyse a value model of the business network raised by the various stakeholders. Once an economically feasible business network is identified, we then take the resulting value model as a starting point and subsequently derive a model that considers the goals of each participant within the business network. These high-level goals are then refined into a set of more specific sub-goals. We believe that the explicit definition of these relations facilitates traceability among goals and the (design) artefacts that ultimately realize the goals. Typically these artefacts are business and IT services, and the processes and rules that support these services. Finally, service orchestration models (e.g., BPEL processes) can be derived from the refined goal model by identifying and articulating the causal relations between the participants' goals. These causal relations provide our basis for the coordination of services that accomplish these goals.

\section{RELATED WORK}

In (Gordijn et al. 2006), the combined use of goal and value models is explored using two requirements engineering techniques, namely $i^{*}$ (Yu 1997) and $\mathrm{e}^{3}$ value (Gordijn \& Akkermans 2001). The main focus lies in showing how the complementary use of both techniques can help in creating, representing, and analyzing e-service business models. E-service models are then used for modelling interaction points of cooperating IT systems, within and between enterprises. In (van der Raadt et al. 2005), the authors propose a method for transforming an $\mathrm{i}^{*}$ goal model into an $\mathrm{e}^{3}$ value value model. First, the method involves modelling the strategic business goals of the enterprises that want to cooperate. It then focuses on formulating alternatives for reaching those goals. Then, for each proposed alternative, an $\mathrm{e}^{3}$ value model is constructed. These value models are then used to evaluate whether those alternatives are economically viable for each of the enterprises involved.

In (Pijpers \& Gordijn 2007), an approach is introduced to arrive at a business process model of a business network that is consistent with an economic value model of the same business network. The 
authors propose a step-wise approach that first considers the independent transfer of the ownership right of a value object and the actual object itself, and then considers time ordering of these transfers. The consistency between models is achieved by applying a set of informal guidelines describing how to move in a structured way from a value model to a process model.

In (Zlatev \& Wombacher 2005), a methodology is proposed for determining the consistency of a process model and a value model, where both models are assumed to represent the same business network but were created independently from each other. In order to determine consistency, a preparation step is carried out in which the given models are 'reduced' such that they only use constructs present in both models. Since the reduced models describe only the common concepts and relations, they can be compared for consistency. The major disadvantage of this approach is that, under certain conditions, it is still possible that the original value model and process model are mutually consistent, while the reduced models show otherwise.

The authors of (Decreus \& Poels 2009) present an approach to generate business process models from enriched goal models. They extend existing $i^{*}$ goal models with semantic annotations in order to describe dynamic constraints among goals and tasks. Then, they propose detailed mappings to translate the semantically enriched goal models into business process models.

In (Koliadis \& Ghose 2006, Koliadis et al. 2006), a methodology is proposed for relating business process models to high-level stakeholder goals. They use BPMN to represent business models and KAOS and $\mathrm{i}^{*}$ to express goals. The relationship between processes and goals is evaluated with informal techniques, however with consideration of dynamic environments in which changes to goals and processes constantly emerge.

\section{FROM VALUES TO GOALS}

\subsection{Example}

We will use a simple running example to illustrate our approach. The example refers to a business network in the Dutch electricity sector, as reported in (Pijpers \& Gordijn 2008).

In the electricity business network, suppliers provide electricity to consumers, and the consumers pay for this electricity. The Suppliers acquire electricity from a set of producers. In this context, consumers, suppliers and producers define different market segments taken into consideration and used to represent a set of actors. For instance, a specific actor may use technical expertise to become the lowcost electricity producer within his company's market segment, while other actors may choose a production strategy that emphasizes flexibility, consumer choice, and quality.

In order to be eligible for electricity provision, a consumer must first obtain power distribution capabilities from a distributor. In practice, a physical infrastructure (consisting of cables and transformers) is needed to transport the electricity, and the consumer also has to pay for this. Instead of collecting money directly from the consumers, the distributor sells these "debts" to the supplier, which, in turn, charges a corresponding fee from the consumers. It is assumed that related participants in this network have pair-wise contracts which are valid for a year. A contract is yearly renewed, unless one of the participants indicates that it does not want to continue the relationship. The new contract may state new conditions on the relationship, such as changed tariffs for the electricity.

\subsection{Value Modelling}

In short, our proposal is to initially design (and evaluate) a value model, e.g. using the $\mathrm{e}^{3}$ value language. The result of this design step for our example is illustrated in Fig. 2.

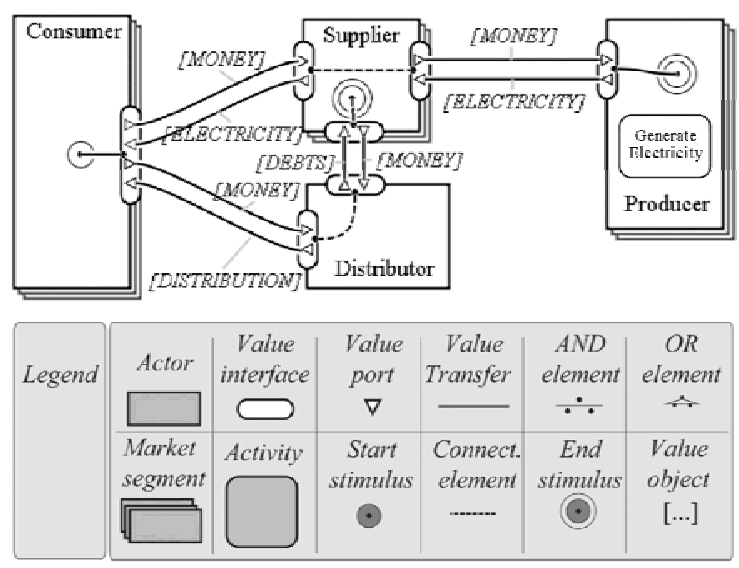

Figure 2: e3value model of Dutch Electricity Sector (adapted from (Pijpers \& Gordijn 2008)).

It is important to realize that value exchanges represented in the value model do not necessarily coincide with physical exchanges. For example, the model explicitly represents that the distributor provides the electricity distribution service to the 
consumer in exchange of some agreed sum of money. Indeed, this is correct from the value perspective. But a naive interpretation of the model that the customer directly pays the distributor is wrong. As already announced in the introduction of the example, the actual money flow is via the supplier.

\subsection{An Intermediate Step}

Since a value transfer in an $\mathrm{e}^{3}$ value model implicitly involves the transfer of both the value object itself and its respective ownership right, it is not possible to directly derive from an $\mathrm{e}^{3}$ value model the actual physical flow of value objects. To this end, we followed the approach described in (Pijpers \& Gordijn 2007) to derive an $\mathrm{e}^{3}$ value (physical) model from the $\mathrm{e}^{3}$ value model, such that the $\mathrm{e}^{3}$ value (physical) model will represent the physical transfer of the value objects rather than the value transfer. In brief, this approach incorporates the physical flow of a value object by distinguishing between the transfer of the ownership right of an object and the transfer of the value object itself (transfer of physical possession). The obtained $\mathrm{e}^{3}$ value (physical) model is shown in Fig. 3.

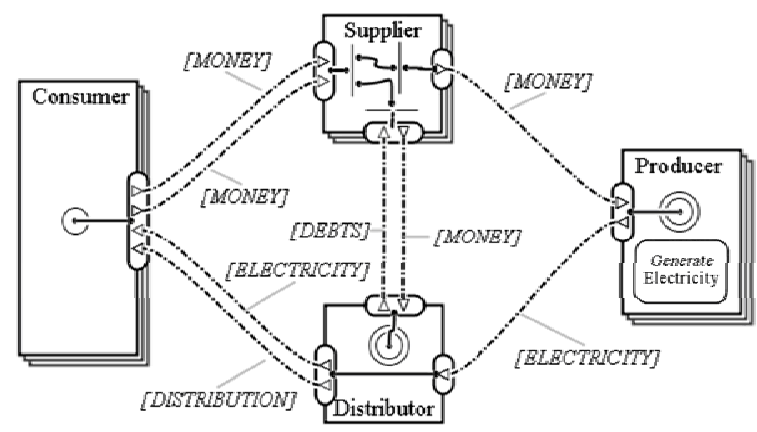

Figure 3: e3value (physical) Model of Dutch Electricity Sector (adapted from (Pijpers \& Gordijn 2008)).

\subsection{Goal Modelling}

In previous work, we have presented a language for modelling requirements in enterprise architectures - namely ARMOR (Quartel et al. 2009), which is aligned with the ArchiMate language (Jonkers et al. 2007) and supports the modelling of goals and requirements during the early and late requirements engineering phases. In this paper, we use the ARMOR language for representing and analysing goal models. ARMOR was inspired by the Business Motivation Model
(OMG 2007), the $i^{*}$ framework (Yu 1997) and KAOS (van Lamsweerde 2008). Table 1 shows the notation for the main abstract language elements.

Table 1: ARMOR notation.

\begin{tabular}{|c|c|}
\hline Abstract element & Concrete notation \\
\hline Stakeholder & Stakeholder $\frac{P}{x}$ \\
\hline Hard goal & Hard goal ()) \\
\hline Soft goal & Softgoal \\
\hline Requirement & Requirement $\square$ ) \\
\hline Realisation & 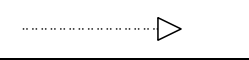 \\
\hline AND realisation & $\triangle D$ \\
\hline OR realisation & 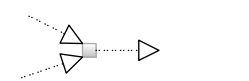 \\
\hline Contribute relation & $+1-$ \\
\hline Conflict relation & 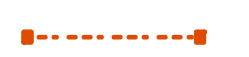 \\
\hline
\end{tabular}

A goal is defined as some desired result that is to be realized in the problem domain. Here a desired result represents some desired situation, state or value, such as a satisfied customer, increase of sales, a completed purchase, the calculation of some exchange rate, the delivery of some goods, etc. Eventually, this result has to be realized through cooperation of the participants in a business network. A distinction can be made between hard and soft goals. This distinction concerns the criteria for assessing the satisfaction of a goal. In case of a hard goal, these criteria are clear and precise. In case of a soft goal, these criteria are typically vague or subject to interpretation. A requirement is a goal that can be assigned to some system (-to-be), such that the system is made responsible for the satisfaction of the goal. A goal can be refined into one or more subgoals, where the following types of refinement are distinguished:

- AND-realization (conjunction): defining one or more sub-goals that must be satisfied all to satisfy the goal;

- OR-realization (disjunction): defining one or more alternative sub-goals of which at least one must be satisfied to satisfy the goal;

- a combination: typically in disjunctive normal form.

A goal G1 may conflict with the satisfaction of another goal G2, where G1 and G2 can only be a 
hard goal. In addition, a goal G1 may contribute to the satisfaction of another goal G2, where G1 and G2 can both be either a hard or a soft goal. Properties of the contribute relation are:

- type: the type of contribution, i.e., positive or negative;

- strength: the strength of the contribution, e.g., weak or strong.

\subsection{Goal Elicitation and Refinement}

A goal model is derived from a given value model by applying the following guidelines (assuming $\mathrm{e}^{3}$ value and ARMOR employed languages):

1) Identification of stakeholders and their primary goals. For each actor and for each market segment present in the value model, we derive a stakeholder in the goal model. Subsequently, for each economic activity and for each value exchange performed by each actor, we derive a primary goal and associate it with its stakeholder. Fig. 4 illustrates this step with our example for the actor/stakeholder 'producer', where the economic activity 'generate electricity' raises a goal with the same name. In addition, the value exchange between producer and supplier raises another goal that promotes the 'exchange of electricity for money'. Since this value exchange matches a pattern that reflects a selling activity (i.e., the exchange of goods for money between stakeholders), we then rename its respective goal to 'sell electricity'. Other common patterns include: advertising, reservation, insourcing, rating, registration, screening, and resource renting. For a more detailed description of value patterns, we refer to (Zlatev 2007).

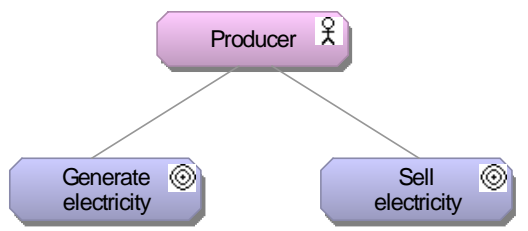

Figure 4: (Step1) Identification of stakeholders and their respective primary goals.

2) Refinement of primary goals. Next, we extend the goal model by refining the primary goals. For this, for each goal derived from a value exchange, we derive sub-goals by analyzing how each value transfer, which constitutes the same value exchange, are physically operated according to the $\mathrm{e}^{3}$ value(physical) model (shown in Fig. 3). This step is illustrated in Fig. 5. The value transfer between producer and supplier raises the goal that promotes the transfer of money from one stakeholder (supplier) to another (producer). Since this value transfer matches a pattern that reflects a charging activity, we then rename its respective goal to 'charge supplyer'. An analogous derivation is carried out for the transfer of electricity between producer and distributor. This transfer raises the goal 'deliver electricity to distributor'.

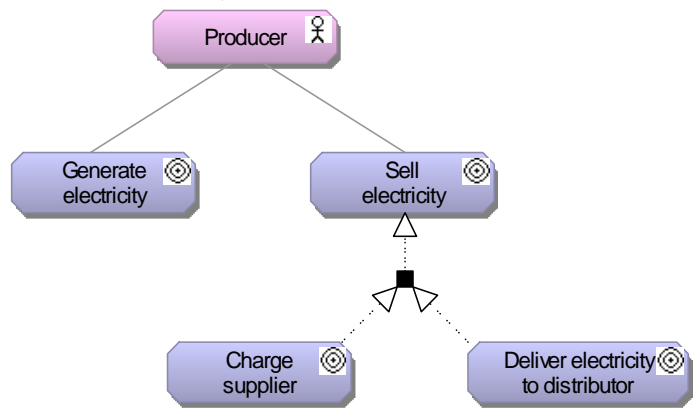

Figure 5: (Step2) Refinement of primary goals into subgoals.

3) Manual refinement of sub-goals. Finally, we extend the goal model by manually refining the goals obtained from steps 1 and 2 into more concrete and specific sub-goals. This is essentially a creative process which requires domain-specific knowledge since the appropriate information can not be directly derived from the given models. However, Fig. 6 illustrates a possible result of this step for our example.

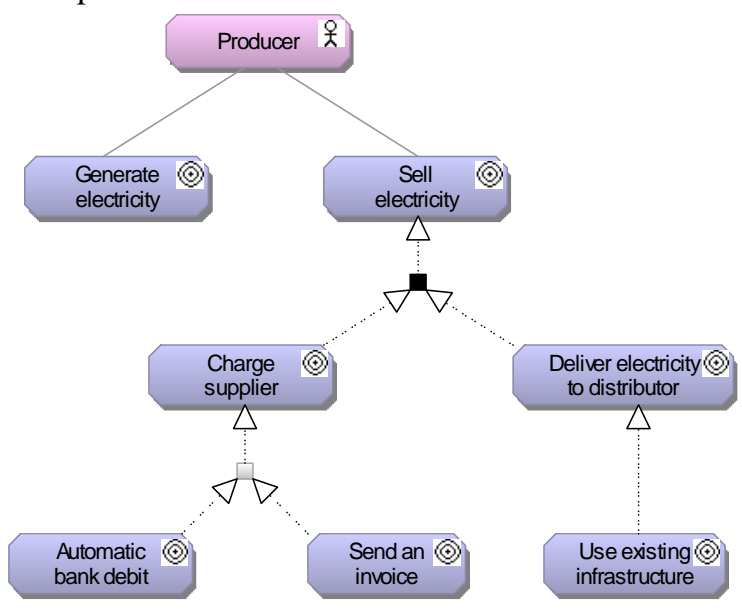

Figure 6: (Step3) Manual refinement of sub-goals.

Fig 7 shows the obtained diagrams for the remaining stakeholders, namely supplier and distributor, after steps 1 and 2. 


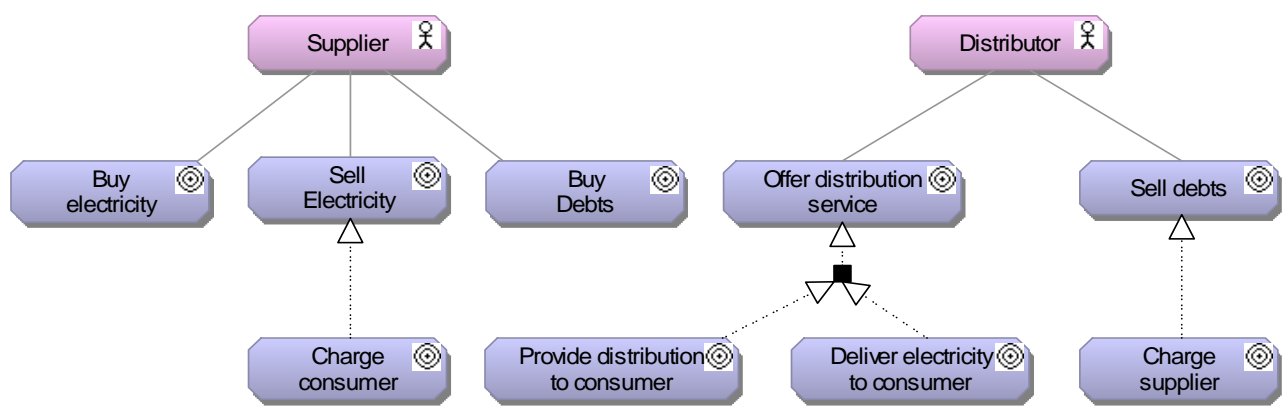

Figure 7: The supplier's and distributor's goals, after steps 1 and 2.

\section{FROM GOALS TO PROCESSES TO SERVICES}

Since goals are intended to be achieved by (intraand inter-) business processes, during late requirements elicitation, functional goals should be mapped onto activities of these processes.

Non-functional requirements, specified as soft goals, are then refined into measurable indicators. Our objective is to finally operationalize goals as ITlevel services which can be rooted in a SOA.

We are still in the first phase of investigating the possibilities for doing this. The following are preliminary ideas, which should be further studied and converted into concrete guidelines:

- Goals can be associated with activities, where the purpose of each activity is to achieve a result that is supportive of, or even fully operationalize the associated goal. Goal refinement may be driven by the objective to directly relate goals to existing IT services. For example, when goals can be formulated in the proper formalism, they may be used as input to goal-based service discovery approaches (Bonino da Silva Santos et al. 2008).

- The goal model can be analyzed for dependencies and relationships between identified goals, in order to find clues on how the associated activities (or services) must be related, such as the time ordering in which activities must take place and the value dependency of one activity on another. For example, if the goal model states that a goal G1 can only be satisfied if G2 and G3 are satisfied, this means that the activities associated with G2 and G3 both have to be executed for the fulfilment of G1, and that the activity associated with G1 (if not already fulfilled by G2 and G3) can only be completed after completion of the activities associated with G2 and G3. The precise implications of goal relationships (conjunction, disjunction, contribute, conflict) for activity relationships need to be further investigated.

- Sets of activities related in this way form abstract business processes. These processes represent behaviour that participants in a business network wish to expose. Therefore they can be interpreted as (part of) an abstract service choreography. Corresponding service orchestrations or missing parts of the service choreography can be created using patterns such as proposed in (Khalaf et al. 2006), or designed and validated using service design frameworks such as (Quartel et al. 2007).

- If for identified abstract services no corresponding existing services can be discovered using (semantic) service discovery approaches, they have to be designed and implemented. This means that manual effort is needed to create WSDL descriptions and to implement the WSDL interfaces and service processes. Similarly, for compositions of services executable processes have to be developed, such as BPEL processes.

\section{CONCLUSION}

With the advent of business networks, the alignment between business and information technology gets a new dimension. In such networks, there is not a single point of authority for making decisions about IT support to solve conflicts in requirements that the participants in these networks may have (Wieringa et al. 2005).

In our approach, a requirement defines a goal that can be assigned to some system (-to-be), such that the system is made responsible for the satisfaction of the goal. In this sense, a requirement should model desired characteristics of products, processes and services under development. The main reason for using goal modelling is that the rationale for designing and changing a system is to be found outside the system itself (Loucopoulos 1994). By explicitly modelling and representing enterprise 
goals, goal-oriented modelling languages help in analysing, changing, and communicating requirements to stakeholders.

By outlining guidelines for deriving intentional goal models from economic value models, we want to facilitate the use of both models during requirements engineering and design of serviceoriented business networks, in order to help stakeholders in considering ways to compose supplier services into bundles that are valuable from a consumer perspective and profitable for all concerned (Wieringa et al. 2005). We believe that value models and goal models are relevant tools that should be explored in this context. The paper offers initial guidelines for deriving a model that considers the goals of each participant within a business network. In this work, we used $\mathrm{e}^{3}$ value for value models ARMOR for goal models.

Our work differs in several aspects from existing work. The difference is mainly that of scope and focus. In contrast with (Zlatev \& Wombacher 2005), our approach introduces goal modelling as an intermediate perspective to arrive at a business process model of a business network. In contrast with (van der Raadt et al. 2005), in the goal analysis, we move the focus from more general strategic goals of organizations to more specific goals of reciprocal cooperation efforts within business networks. These goals and their refinements into architectural requirements form the basis of our approach.

As future work we intend to identify further guidelines, investigate the formalization of the approach, the resolution of conflicts between goals, the late requirements phase, and the automation of steps 1 and 2 (of the goal elicitation and refinement phase). In order to validate our approach, we want to consider further scenarios and use cases where goal interdependencies and value exchanges require strategic thinking and conflict resolution. To be economically and operationally viable for business use, our approach would benefit from additional analysis techniques (e.g. risk and value analysis).

\section{ACKNOWLEDGEMENTS}

This work is part of the Freeband A-MUSE project (http://a-muse .freeband.nl), which is supported by the Dutch government under contract BSIK 03025.

\section{REFERENCES}

Bonino da Silva Santos, L.O., Ferreira Pires, L., van Sinderen, M.J., 2008. A goal-based framework for dynamic service discovery and composition. In ACT4SOC 2008, 2nd International Workshop on Architectures, Concepts and Technologies for Service Oriented Computing. INSTICC Press, pp. 67-78.

Decreus, K., Poels, G., 2009. Mapping semantically enriched Formal Tropos to business process models. In SAC 2009, 24th Annual ACM Symposium on Applied Computing. ACM, pp. 371-376.

Dijkman, R.M., Quartel, D.A.C., van Sinderen, M.J., 2008. Consistency in multi-viewpoint design of enterprise information systems. Information and Software Technology, 50 (7-8): 737-752.

Gordijn J., Akkermans H., 2001. E3-value: design and evaluation of e-business models. IEEE Intelligent Systems, 16(4):11-17.

Gordijn, J., Yu, E., van der Raadt, B. 2006.. E-service design using $i^{*}$ and e3value modeling. IEEE Software, 23(3): 26-33.

Jonkers, H. et al., 2007. Concepts for architectural description, Technical Report TI/RS/2003/007, Enschede: Telematica Instituut.

Khalaf, R., Keller, A., Leymann, F., 2006. Business processes for web services: principles and applications. IBM Systems Journal, 45(2): 425-446.

Koliadis, G., Ghose, A., 2006. Relating business process models to goal-oriented requirements models in KAOS. In PLAW 2006, Pacific Rim Knowledge Acquisition Workshop. LNCS 4303, Springer, pp. 2539.

Koliadis, G. et al., 2006. Combining i* and BPMN for business process model lifecycle management. In BPM 2006 Workshops, Business Process Management Workshops. LNCS 4103, Springer, pp. 416-427.

Loucopoulos, P. (1994) The F3 (from fuzzy to formal) view of requirements engineering. Journal of Ingenierie des Systemes d'Information, 2(6): 639-655.

Luthria, H., Rabhi, F., 2009. Service oriented computing in practice - an agenda for research into the factors influencing the organizational adoption of service oriented architectures. Journal of Theoretical and Applied Electronic Commerce Research, 4(1): 39-56.

Mantovaneli Pessoa, R., Quartel, D.A.C., van Sinderen, M.J., 2008. A comparison of data and process mediation approaches. In I-WEST 2008, 2nd Workshop on Enterprise Systems and Technology. INSTICC Press, pp. 48-63.

OASIS, 2006. Reference Model for Service Oriented Architecture 1.0. URL http://www.oasisopen.org /committees/download.php/19361/soa-rm-cs.pdf.

OMG, 2007. Business Motivation Model (BMM) Specification. Object Management Group. URL http://www.omg.org/

Papazoglou, M. Ribbers, P., 2006. e-Business: organizational and technical foundations. John Wiley\& Sons Inc.

Pijpers, V., Gordijn, J., 2007. Bridging business value models and process models in aviation value webs via possession rights. In HICCS 2007, 40th Annual Hawaii international Conference on System Sciences. IEEE Computer Society. 
Pijpers, V., Gordijn, J., 2008. Consistency checking between value models and process models: a best-ofbreed approach. In BUSITAL 2008, 3rd International Workshop on Business/IT Alignment and Interoperability. CEUR-WS.org , pp. 58-72.

Quartel, D.A.C., Steen, M.W.A., Pokraev, S.V., van Sinderen, M.J., 2007. COSMO: a conceptual framework for service modelling and refinement. Information Systems Frontiers, 9 (2-3): 225-244.

Quartel, D.A.C., Pokraev, S.V., Mantovaneli Pessoa, R., van Sinderen, M.J., 2008. Model-driven development of a mediation service. In EDOC 2008, 12th International IEEE Enterprise Distributed Object Computing Conference. IEEE Computer Society, pp. 117-126.

Quartel, D., Jonkers, H., Engelsman, W., 2009. A requirements modelling language for enterprise architecture: ARMOR for requirements modelling. Enschede: Telematica Instituut.

Tapscott, D., Ticoll, D., Lowy, A., 2000. Digital capital: harnessing the power of business webs. Ubiquity, 1(13): 3.

van der Raadt, B., Gordijn, J., Yu, E., 2005. Exploring web services from a business value perspective. In $R E$ 2005, 13th IEEE International Conference on Requirements Engineering. IEEE Computer Society, pp. 53-62.

van Lamsweerde, A., 2008. Requirements engineering: from craft to discipline. In FSE 2008, 16th ACM Sigsoft Intl. Symposium on the Foundations of Software Engineering. ACM, pp. 238-249.

Wieringa, R.J., Gordijn, J., van Eck, P.A.T., 2005. Valuebased business-IT alignment in networked constellations of enterprises. In REBNITA 2005, 1st International Workshop on Requirements Engineering for Business Need and IT Alignment. Univ. of New South Wales Press, pp. 38-43.

Yu, E., 1997. Towards modelling and reasoning support for early-phase requirements engineering. In ISRE '97, 3rd IEEE Int. Symp. on Requirements Engineering. IEEE Computer Society, pp. 226-235.

Zlatev, Z., Wombacher, A., 2005. Consistency between $\mathrm{e}^{3}$ value models and activity diagrams in a multiperspective development method. In CoopIS 2005, COopIS, DOA, and ODBASE: OTM Confederated International Conferences, On the Move to Meaningful Internet Systems. LNCS 3760, Springer, pp. 520-538.

Zlatev, Z.V., 2007. Goal-oriented design of value and process models from patterns. PhD thesis, CTIT Ph.D.-thesis series No.07-102, Enschede: University of Twente. 